\title{
The generalized force-velocity relationship explains why the preferred pedaling rate of cyclists exceeds the most efficient one
}

Journal Article

Author(s):

Kohler, Götz; Boutellier, Urs

Publication date:

2005-05

Permanent link:

https://doi.org/10.3929/ethz-b-000032877

Rights / license:

In Copyright - Non-Commercial Use Permitted

Originally published in:

European Journal of Applied Physiology 94(1-2), https://doi.org/10.1007/s00421-004-1283-2 
Götz Kohler · Urs Boutellier

\section{The generalized force-velocity relationship explains why the preferred pedaling rate of cyclists exceeds the most efficient one}

Accepted: 22 November 2004 / Published online: 19 January 2005

(C) Springer-Verlag 2005

\begin{abstract}
The most efficient pedaling rate (lowest oxygen consumption) at a workload of $50-300 \mathrm{~W}$ has been reported to be in the range of $42-60 \mathrm{rpm}$. By contrast, most competitive cyclists prefer a pedaling rate of more than $90 \mathrm{rpm}$. The reason for this difference is still unknown. We assume that the high pedaling rate preferred by cyclists can be explained by the inherent properties of muscle fibers. To obtain statements which do not depend on muscle's cross-section and length, we generalized Hill's characteristic equations where muscle force and heat liberation are related to shortening velocity. A pedaling rate of $f_{\eta \max }$ yields to maximal efficiency, whereas the higher pedaling rate $f_{P \max }$ leads to maximal power. The ratio $f_{P \max } / f_{\eta \max }$ between these two pedaling rates ranges from 1.7 to 2.4 , and it depends on the muscle's fiber-type composition. In sprints and competitions of very short duration, $f_{P \max }$ is more advantageous because energy supply is not the predominant limiting factor. The price to be paid for the most powerful pedaling rate is lower efficiency and higher energy cost. In longer exercises, economy is more important and the optimal pedaling rate shifts toward $f_{\eta \max }$. We conclude that the optimal pedaling rate, representing the fastest race performance, is not fixed but depends on race duration; it ranges between $f_{\eta \max }$ and $f_{P \max }$. Our results are not only of interest for competitive cyclists but also for investigations using cycle ergometers:
\end{abstract}

This revised version was published online in March 2005 with corrections to the equations.

G. Kohler $(\bowtie)$

Biophysical Chemistry, Biocenter of the University, University of Basel, Basel, Switzerland

E-mail: goetz.kohler@unibas.ch

Tel.: + 41-61-2672196

Fax: $+41-61-2672189$

U. Boutellier

Exercise Physiology, Institute for Human Movement Sciences,

Swiss Federal Institute of Technology, Zurich, Switzerland

U. Boutellier

Institute of Physiology, University of Zurich, Zurich, Switzerland maximum power might not be reached by using a pedaling rate near the most efficient one.

Keywords Force $\cdot$ Power Efficiency $\cdot$ Optimal pedaling rate $\cdot$ Cadence

\section{Introduction}

The most efficient pedaling rate (lowest oxygen consumption) at a workload of $50-300 \mathrm{~W}$ is in the range of 42-60 rpm (di Prampero 2000). By contrast, field observations indicate that most competitive cyclists prefer pedaling rates above $90 \mathrm{rpm}$. In short track races, pedaling rates may even exceed $120 \mathrm{rpm}$. How can this contradiction between field and laboratory observations be explained? Hemodynamics may affect the preferred pedaling rate, because a high pedaling rate can result in a more effective action of the skeletal-muscle pump (Gotshall et al. 1996). Another suggestion is that the internal work-rate component (needed for moving the limbs) is proportionally smaller at pedaling rates between 82 and $101 \mathrm{rpm}$, resulting in greater mechanical efficiency (Widrick et al. 1992). In our opinion, no convincing suggestion has yet been advanced that could explain this "cadence paradox".

The actual goal of a competitive cyclist is to win a race, and winning depends on achieving the greatest power output over a given distance (less frequently over a given time period). The greatest power output is produced at pedaling rates between 80 and $120 \mathrm{rpm}$ (Baron 2001; Sargeant et al. 1981). We avoid the term "optimal pedaling rate" because it has been used with different meanings: di Prampero (2000) identifies it with the most efficient pedaling rate, whereas other authors use the term for the pedaling rate producing the greatest power (Baron 2001; Dorel et al. 2003; Martin and Spirduso 2001) or the cadence producing the lowest EMG amplitude for a given power (Macintosh et al. 2000). 
We propose that the preferred pedaling rate of competitive cyclists can be explained by the inherent properties of muscle fibers that are described by Hill $(1938,1964)$ as functions relating muscle force and heat liberation to shortening velocity (hereafter, we will name these functions force-velocity relationship and heat liberation; formulas are given in Calculations). These functions contain constants that depend on muscle size and fiber type. Thus, each muscle has its own force-velocity relationship and its own heat liberation. Therefore, statements based on these relationships are valid only for a given muscle. In the following, we shall transform the force-velocity relationship and the heat liberation into general terms where constants depending on muscle size are eliminated. Statements based on these new general equations apply to any muscle independent of its size. These theoretical considerations add a new dimension to the question why the preferred pedaling rate of cyclists exceeds the most efficient one.

\section{Calculations}

The general force-velocity relationship

Hill (1938) found the following force-velocity relationship for concentric contractions:

$F=b\left(F_{0}+a\right) /(v+b)-a$

where $F$ is the force, $F_{0}$ is the maximal isometric force, $v$ is the velocity of fiber shortening, $a$ is a constant depending the cross-sectional area of the muscle, and $b$ is a constant depending on the length of the muscle.

The force-velocity relationship was found in vitro in isolated muscle preparations of a single fiber-type. Human skeletal muscles, however, are composed of two main types of muscle fibers. As the two main fiber types have different contractile properties, the constants $a$ and $b$ depend on the fiber type. Thus, Eq. 1 is not valid for mixed muscles composed of both fiber types. The $F$ developed by a given mixed muscle, at a given $v$, is the sum of the $F$ values developed by both groups of fibers:

$$
\begin{aligned}
F= & F_{\mathrm{I}}+F_{\mathrm{II}} \\
F= & b_{\mathrm{I}}\left(F_{0, \mathrm{I}}+a_{\mathrm{I}}\right) /\left(v+b_{\mathrm{I}}\right)-a_{\mathrm{I}} \\
& +b_{\mathrm{II}}\left(F_{0, \mathrm{II}}+a_{\mathrm{II}}\right) /\left(v+b_{\mathrm{II}}\right)-a_{\mathrm{II}}
\end{aligned}
$$

The subscripts I and II are used to indicate that each fiber type is characterized by its own specific constants $a$, $b$, and $F_{0}$. All these specific constants must be eliminated in order to obtain a force-velocity relationship that does not depend on the size of the muscle. We will first set out to eliminate the two constants $a_{\mathrm{I}}$ and $a_{\mathrm{II}}$. The ratio $a_{0}=a / F_{0}$ is similar for any muscle of a single fiber-type (Hill 1938). Therefore, $a_{\mathrm{I}}$ and $a_{\mathrm{II}}$ can be replaced by:

$$
\begin{aligned}
& a_{\mathrm{I}}=a_{0, \mathrm{I}} F_{0, \mathrm{I}} \\
& a_{\mathrm{II}}=a_{0, \mathrm{II}} F_{0, \mathrm{II}}
\end{aligned}
$$

Faulkner et al. (1986) determined that $a_{0, \mathrm{I}}=a_{\mathrm{I}} / F_{0, \mathrm{I}}=$ 0.15 for type I fibers of human muscle, and that $a_{0, \mathrm{II}}=a_{\mathrm{II}} / F_{0, \mathrm{II}}=0.25$ for type II fibers.

Inserting Eq. $3 \mathrm{a}$ and $3 \mathrm{~b}$ in Eq. 2, and dividing by the isometric force $F_{0}=F_{0, \mathrm{I}}+F_{0, \text { II }}$ of a mixed muscle, yields the $F$ related to the isometric force.

$$
\begin{aligned}
F / F_{0}= & F_{0, \mathrm{I}}\left[b_{\mathrm{I}}\left(1+a_{0, \mathrm{I}}\right) /\left(v+b_{\mathrm{I}}\right)-a_{0, \mathrm{I}}\right] / F_{0} \\
& +F_{0, \mathrm{II}}\left[b_{\mathrm{II}}\left(1+a_{0, \mathrm{II}}\right) /\left(v+b_{\mathrm{II}}\right)-a_{0, \mathrm{II}}\right] / F_{0}
\end{aligned}
$$

Subsequently $F_{0, \text { I }}$ and $F_{0, \text { II }}$ are removed. As both fiber types have similar capacities for generating isometric force (Faulkner et al. 1986), the ratio $F_{0, \mathrm{I}} / F_{0}$ is equal to the ratio between the cross-sectional area of fiber type I and the cross-sectional area of the whole muscle. The same applies to fiber type II. Defining the fiber-type composition factor $x=$ (cross-sectional area of type II fibers/cross-sectional area of the whole muscle), Eq. 4a can be written as:

$$
\begin{aligned}
F / F_{0}= & (1-x)\left[b_{\mathrm{I}}\left(1+a_{0, \mathrm{I}}\right) /\left(v+b_{\mathrm{I}}\right)-a_{0, \mathrm{I}}\right] \\
& +x\left[b_{\mathrm{II}}\left(1+a_{0, \mathrm{II}}\right) /\left(v+b_{\mathrm{II}}\right)-a_{0, \mathrm{II}}\right]
\end{aligned}
$$

The constants $b_{\mathrm{I}}$ and $b_{\mathrm{II}}$ will now be eliminated as follows. Figure 1 shows the force-velocity relationship for type I $(x=0)$ and type II (and $x=1)$ fibers. By setting the $F$ to zero in Eq. 1, we obtain the maximum shortening velocities, $v_{\max , \mathrm{I}}=b_{\mathrm{I}} / a_{0, \mathrm{I}}\left(\right.$ for $x=0$ ), and $v_{\max , \mathrm{II}}$ $=b_{\mathrm{II}} / a_{0, \mathrm{II}}($ for $x=1)$. This allows us to replace $b_{\mathrm{I}}$ and $b_{\mathrm{II}}$ as follows:

$b_{\mathrm{I}}=a_{0, \mathrm{I}} v_{\max , \mathrm{I}}$
$b_{\mathrm{II}}=a_{0, \mathrm{II}} v_{\max , \mathrm{II}}$

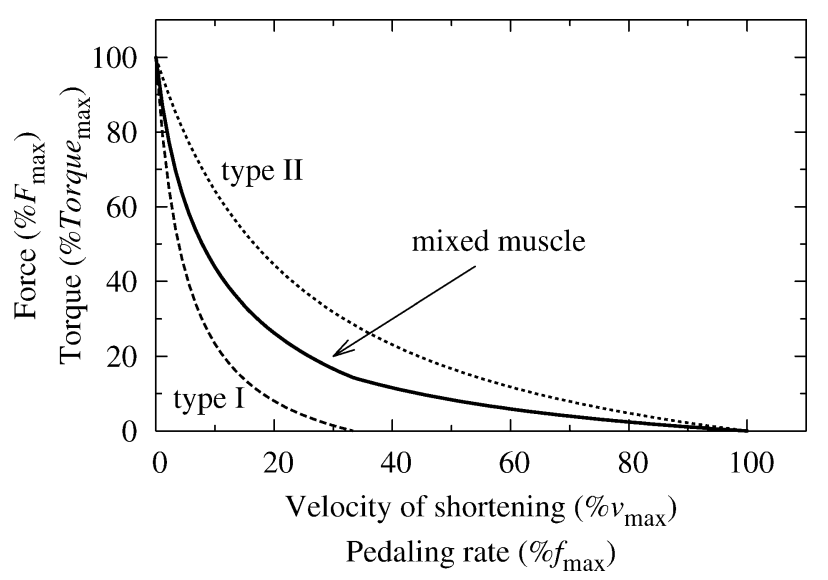

Fig. 1 General force-velocity relationships, which apply to muscles with any cross-sections and lengths. Dashed line (type I) A hypothetical muscle composed exclusively of type I fibers; dotted line (type II) a muscle composed exclusively of type II fibers. Continuous line A muscle with equal cross-sectional area of both fiber types (50\% type I, $50 \%$ type II), so that in Eq. $2 \mathrm{~b}, x=0.5$. Muscle shortening velocity is proportional to pedaling rate (see text). Therefore in this diagram, as well as the subsequent ones, when appropriate, shortening velocity can be replaced by pedaling rate 
In human muscle, the maximum shortening velocity of type II fibers is three times that of type I fibers (Faulkner et al. 1986). To keep our equations as general as possible, however, we define $V=v_{\max , \text { II }} / v_{\max , \text { I }}$, which allows us to alter the ratio $v_{\max , \text { II }} / v_{\max , \mathrm{I}}$ in case new evidence emerges. Now $b_{\mathrm{I}}$ can also be replaced by:

$b_{\mathrm{I}}=a_{0, \mathrm{I}} v_{\max , \mathrm{II}} / V$

Defining the relative shortening velocity $u=v / v_{\text {max,II }}$ we obtain:

$v=u v_{\max , \mathrm{II}}$

Inserting Eqs. 5b and 5c and 6 in Eq. 4b, we obtain the general force-velocity relationship for mixed muscles which does not contain any muscle-size-dependent constants:

$$
\begin{aligned}
F / F_{0}= & k=(1-x)\left[\left(a_{0, \mathrm{I}}+a_{0, \mathrm{I}}^{2}\right) /\left(u V+a_{0, \mathrm{I}}\right)-a_{0, \mathrm{I}}\right] \\
& +x\left[\left(a_{0, \mathrm{II}}+a_{0, \mathrm{II}}^{2}\right) /\left(u+a_{0, \mathrm{II}}\right)-a_{0, \mathrm{II}}\right]
\end{aligned}
$$

where $k\left(=F / F_{0}\right)$ is the force relative to $F_{0}$. Thus, from Eq. 7 , if $F_{0}$ is known, the force-velocity relationship can be obtained for any given muscle:

$F=F_{0} k$

It should be noted that Eq. 7 is valid only for shortening velocities equal or below $v_{\max , \mathrm{I}}=v_{\max , \mathrm{II}} / V$. At higher velocities, type I fibers do not produce any force (see Fig. 1) and the general force-velocity relationship for mixed muscles is: $k=x\left[\left(a_{0, \mathrm{II}}+a_{0, \mathrm{II}}^{2}\right) /\left(u+a_{0, \mathrm{II}}\right)\right.$ $\left.-a_{0, \mathrm{II}}\right]$

The general power-velocity relationship

Mechanical power is defined as:

$P=F \mathrm{~d} s / \mathrm{d} t=F v$

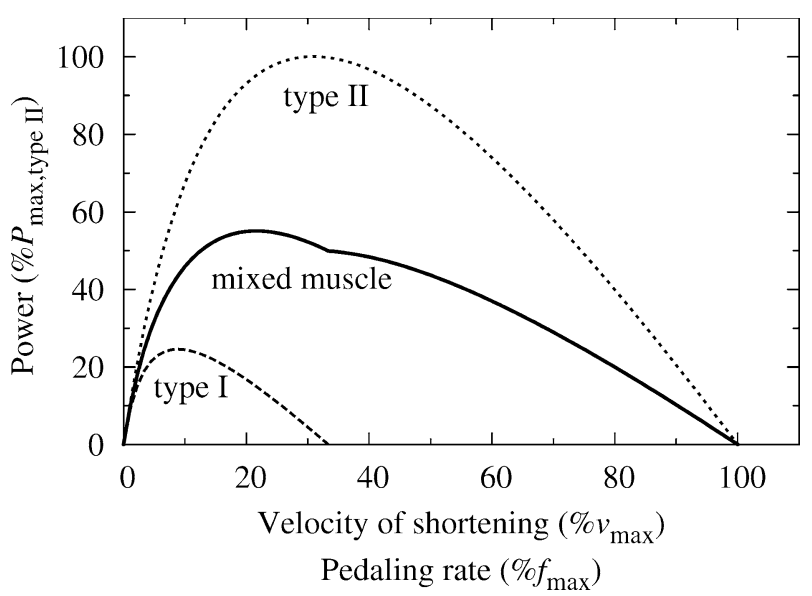

Fig. 2 General power velocity (or power-pedaling-rate) relationship. For symbols and definitions see Fig. 1
Inserting Eqs. 6, 8 and 9, we obtain the following power-velocity relationship for a given muscle:

$P=F_{0} k u v_{\max , \text { II }}$

Dividing Eq. 10 by $F_{0}$ and $v_{\text {max,II }}$ yields the general power-velocity relationship $p=P /\left(F_{0} v_{\max , \text { II }}\right)$, which does not include any size- or fiber-type-dependent constants:

$p=k u$

Figure 2 shows the general power-velocity relationship for both fiber types. The velocity of shortening that produces the greatest power can be found by setting the derivative of Eq. 11 equal to zero. For pure fiber types, the most powerful shortening velocities are given by the equations:

$u_{P \max , \mathrm{I}}=\left[\left(a_{0, \mathrm{I}}+a_{0, \mathrm{I}}^{2}\right)^{1 / 2}-a_{0, \mathrm{I}}\right] / V$ and

$u_{P \max , \mathrm{II}}=\left(a_{0, \mathrm{II}}+a_{0, \mathrm{II}}^{2}\right)^{1 / 2}-a_{0, \mathrm{II}}$

It is difficult to arrive at an analytical solution for this maximum problem for mixed muscles $(x \neq 0,1)$. Therefore, we used numerical calculus to obtain the most powerful shortening velocity: Power was calculated for a given fiber-type proportion while varying the shortening velocities. We used a shortening velocity step width of $0.1 \%$. From Fig. 3, we can see that the most powerful shortening velocity increases as the fiber type II proportion increases.

The general efficiency-velocity relationship

Mechanical efficiency is defined as:

$\eta=P /(P+\mathrm{d} Q / \mathrm{d} t)$

where the second term of the denominator is the rate of heat liberation from the muscle. This $\mathrm{d} Q / \mathrm{d} t$ has

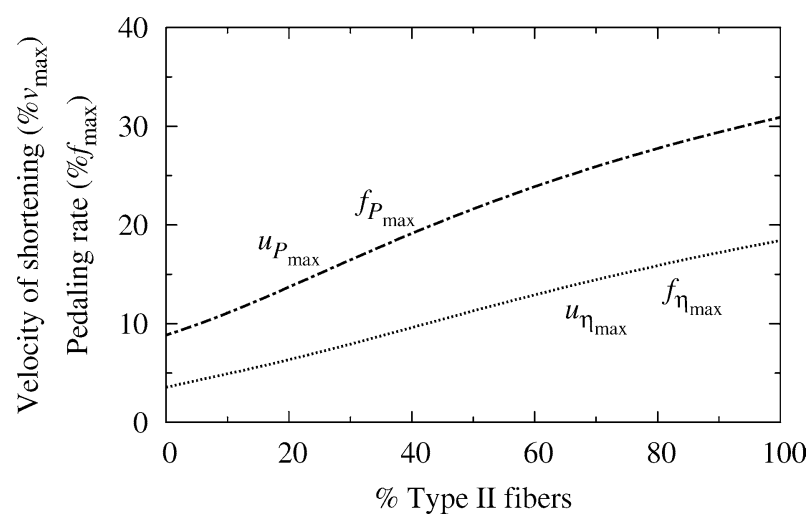

Fig. 3 The most powerful $\left(u_{P \max }\right)$ and the most efficient $\left(u_{\eta \max }\right)$ shortening velocity (or pedaling rate, $f_{P \max }$ and $f_{\eta \max }$ ) as a function of the relative cross-sectional area of type II fibers 
several components: the rate of maintenance heat $(0.987 a b)$ and the rate of shortening heat $[(0.16 \mathrm{~F}$ $\left.+0.18 F_{0}\right) v$ ] (Hill 1964). The constants $a$ and $b$ are the same as in the force-velocity relationship Eq. 1. However, the sum of maintenance heat and shortening heat refers to the initial heat only. It represents the heat released by the initial processes of muscle contraction, ATP and phosphocreatine (PC) splitting. An additional amount of heat (recovery heat) is produced by the resynthesis of ATP and PC. The recovery heat must be proportional to the initial heat because the same quantity of ATP and PC that was split has to be resynthesized. Therefore, the total heat liberation of a muscle is:

$\mathrm{d} Q / \mathrm{d} t=z\left[0.987 a b+\left(0.16 F+0.18 F_{0}\right) v\right]$

where $z$ is not far from a value of 2 (Wendt and Gibbs 1979). The overall heat liberation of a mixed muscle is the sum of the heat liberation of both fiber type fractions:

$$
\begin{aligned}
\mathrm{d} Q / \mathrm{d} t= & (\mathrm{d} Q / \mathrm{d} t)_{\mathrm{I}}+(\mathrm{d} Q / \mathrm{d} t)_{\mathrm{II}} \\
\mathrm{d} Q / \mathrm{d} t= & z\left(0.987 a_{\mathrm{I}} b_{\mathrm{I}}+\left\{0.16\left[b_{\mathrm{I}}\left(F_{0, \mathrm{I}}+a_{\mathrm{I}}\right) /\left(v+b_{\mathrm{I}}\right)-a_{\mathrm{I}}\right]\right.\right. \\
& \left.+0.18 F_{0, \mathrm{I}}\right\} v+0.987 a_{\mathrm{II}} b_{\mathrm{II}} \\
& +\left\{0.16 d\left[b_{\mathrm{II}}\left(F_{0, \mathrm{II}}+a_{\mathrm{II}}\right) /\left(v+b_{\mathrm{II}}\right)-a_{\mathrm{II}}\right]\right. \\
& \left.\left.+0.18 F_{0, \mathrm{II}}\right\} v\right)
\end{aligned}
$$

Inserting Eqs. $3 \mathrm{a}$ and $3 \mathrm{~b}$, and $5 \mathrm{~b}$ and $5 \mathrm{c}$, into Eq. 14, dividing by $z, F_{0}$, and $v_{\max , \mathrm{II}}$, and using the fiber-type composition factor $x$ yields the general heat liberation $h=\mathrm{d} Q / \mathrm{d} t / z F_{0} v_{\max , \text { II }}$ of a mixed muscle:

$$
\begin{aligned}
h= & \mathrm{d} Q / \mathrm{d} t /\left(z F_{0} v_{\max , \mathrm{II}}\right)=(1-x)\left(0.987 a_{\mathrm{I}, 0}^{2} / V\right. \\
+ & \left.\left\{0.16\left[\left(a_{0, \mathrm{I}}+a_{0, \mathrm{I}}^{2}\right) /\left(u V+a_{0, \mathrm{I}}\right)-a_{0, \mathrm{I}}\right]+0.18\right\} u V\right) \\
+ & x\left(0.987 a_{0, \mathrm{II}}^{2}+\left\{0 . 1 6 \left[\left(a_{0, \mathrm{II}}+a_{0, \mathrm{II}}^{2}\right) /\right.\right.\right. \\
& \left.\left.\left.\left(u+a_{0, \mathrm{II}}\right)-a_{0, \mathrm{II}}\right]+0.18\right\} u\right)
\end{aligned}
$$

With this notation, the overall rate of heat liberation for a given muscle can be written as:

$\mathrm{d} Q / \mathrm{d} t=h z F_{0} v_{\max , I I}$

Inserting Eqs. 10 and 16 into Eq. 12, we obtain the general efficiency-velocity relationship for mixed muscles:

$$
\begin{aligned}
\eta & =p F_{0} v_{\max , \mathrm{II}} /\left(p F_{0} v_{\max , \mathrm{II}}+z h F_{0} v_{\max , \mathrm{II}}\right) \\
& =p /(p+z h)
\end{aligned}
$$

The graphs of the general efficiency-velocity relationship for both fiber types are shown in Fig. 4 $(z=2)$. To obtain the most efficient shortening velocity, the derivative $\mathrm{d} \eta / \mathrm{d} u$ must be set equal to zero:

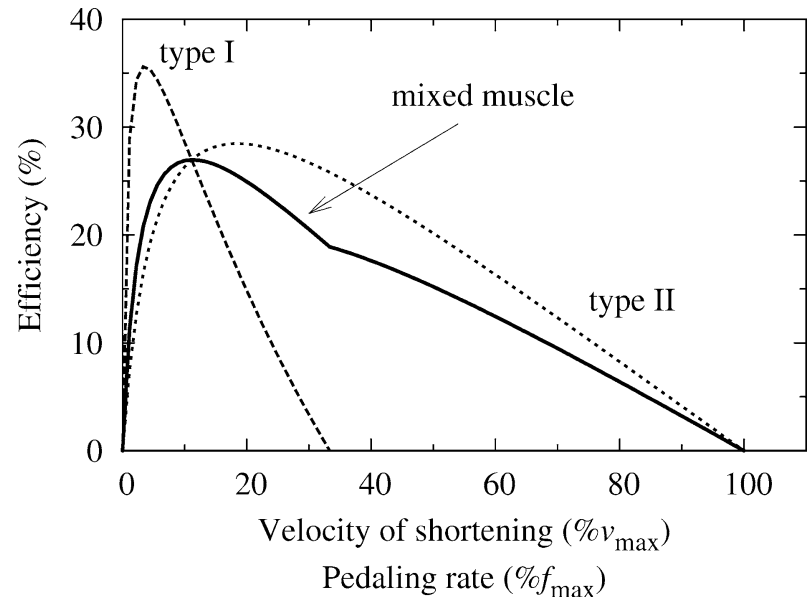

Fig. 4 General efficiency-velocity (or efficiency-pedaling-rate) relationship. For symbols see Fig. 1

$$
\begin{aligned}
0 & =\mathrm{d} \eta / \mathrm{d} u \\
& =(\mathrm{d} p / \mathrm{d} u) /(p+z h)-p(\mathrm{~d} p / \mathrm{d} u+z \mathrm{~d} h / \mathrm{d} u) /(p+z h)^{2} \\
& =(\mathrm{d} p / \mathrm{d} u)(p+z h)-p(\mathrm{~d} p / \mathrm{d} u+z \mathrm{~d} h / \mathrm{d} u) \\
& =p \mathrm{~d} p / \mathrm{d} u+z h(\mathrm{~d} p / \mathrm{d} u)-p \mathrm{~d} p / \mathrm{d} u-z p \mathrm{~d} h / \mathrm{d} u \\
& =z h(\mathrm{~d} p / \mathrm{d} u)-z p \mathrm{~d} h / \mathrm{d} u \\
& =h \mathrm{~d} p / \mathrm{d} u-p \mathrm{~d} h / \mathrm{d} u
\end{aligned}
$$

As Eq. 18 no longer contains the recovery heat $z$, it is possible to calculate the most efficient shortening velocity even if the amount of recovery heat is not known. In particular, Eq. 18 shows that every process which is proportional to ATP and PC splitting, but which is not included in mechanical power, does not affect the most efficient shortening velocity. It is rather difficult to arrive at an analytical solution of Eq. 18, even for a single fiber-type $(x=0$ or $x=1)$. To obtain the most efficient shortening velocity, we used the same numerical calculus that we used previously to determine the shortening velocity that produces the greatest power: Efficiency was calculated for a given fiber-type proportion while

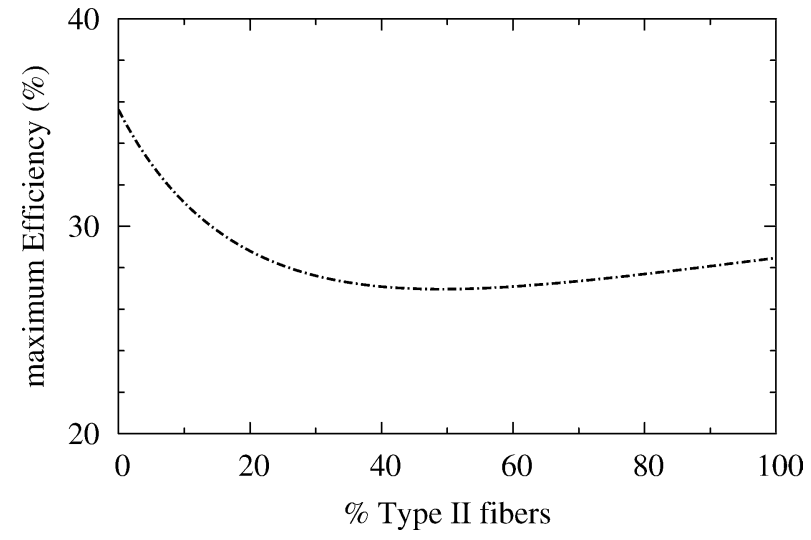

Fig. 5 Maximum efficiency for varying relative fiber-type crosssectional areas 
varying the shortening velocities. We used a shortening velocity step width of $0.1 \%$. Figure 3 shows the most efficient shortening velocity as a function of the musclefiber-type composition. The greater the fiber-type-II proportion, the higher is the most efficient shortening velocity. Furthermore, we can see that the most efficient shortening velocity is lower than the most powerful one for any fiber type ratio. Figure 5 shows the peak efficiency at varying fiber-type proportions.

The most powerful and the most efficient pedaling rate

The calculations performed so far apply to muscle shortening velocities. We shall now attempt to connect the muscle shortening velocities with pedaling rates. As cycling is a movement involving several muscle groups and joints, an exact biomechanical description is highly complex. We assume that it is an adequate approximation to describe cycling as a one-muscle movement. If a cyclist does not alter his position on the bicycle, the velocity of muscle shortening must be proportional to the pedaling rate. In this case, the force-velocity relationship is equivalent to the torque-pedaling-rate relationship, the power-velocity relationship is equivalent to the power-pedaling-rate relationship, and the efficiency-velocity relationship is equivalent to the efficiency-pedaling-rate relationship. Therefore, the ratio between the pedaling rate producing the greatest power $\left(f_{P \max }\right)$ and the most efficient pedaling rate $\left(f_{\eta \max }\right)$ is equal to the ratio between the most powerful velocity of muscle shortening $\left(u_{P \max }\right)$ and the most efficient velocity of muscle shortening $\left(u_{\eta \max }\right)$ :

$f_{P \max } / f_{\eta \max }=u_{P \max } / u_{\eta \max }$

Once the most efficient pedaling rate has been determined in the laboratory, one obtains the most powerful pedaling rate by rearranging Eq. 19:

$f_{P \max }=f_{\eta \max } u_{P \max } / u_{\eta \max }$

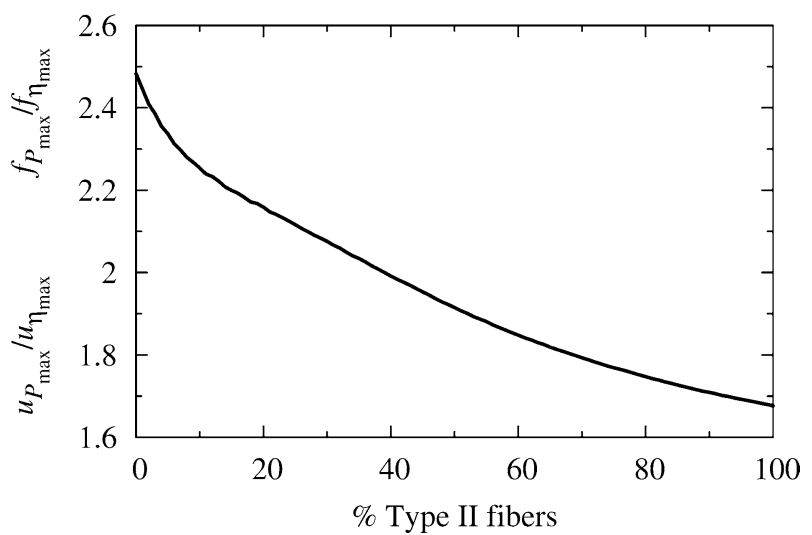

Fig. 6 Relationship between $u_{P \max }$ and $u_{\eta \max }$ for varying relative fiber-type cross-sectional areas. As the muscle shortening velocity is proportional to the pedaling rate, the diagram is in principle also valid for pedaling rates (ratio $f_{P \max } / f_{\eta \max }$ ) and therefore, one can obtain the ratio $u_{\mathrm{P} \max } / u_{\eta \max }$ as a function of the fiber-type composition of the muscle; this is shown in Fig. 6.

\section{Discussion}

We have shown that Hill's characteristic force-velocity and heat-release relationships can be transformed into general equations that exclude any muscle-size and fibertype-dependent factors. Mathematical treatment of these general relationships shows that one pedaling rate yields the greatest efficiency, and another higher rate yields the greatest power. The ratio between these two pedaling rates ranges from 1.68 to 2.44 depending on the fiber-type composition (Fig. 6). If the type I fibers and the type II fibers are equal in cross-sectional area, the most powerful pedaling rate is 1.91 times the most efficient pedaling rate. The most efficient pedaling rate has been determined in the laboratory to be $42-60 \mathrm{rpm}$ in the range of 50-300 W (di Prampero 2000). According to our calculation, the most powerful pedaling rate in this workload range is between 80 and $115 \mathrm{rpm}$ if the cross-sectional areas of both fiber types are equal. This result agrees with laboratory observations where the most powerful pedaling rate has been measured as 80 $120 \mathrm{rpm}$ (Baron 2001; Sargeant et al. 1981).

It should be noted that using the most powerful pedaling rate is not a synonym for maximal power output. Using the most powerful cadence means that the greatest power is produced for a given cross-sectional area. In other words: using the most powerful pedaling rate, a given power can be produced by activating the least number of muscle fibers. The less muscle fibers are used, the lower the resulting EMG activity. The lowest

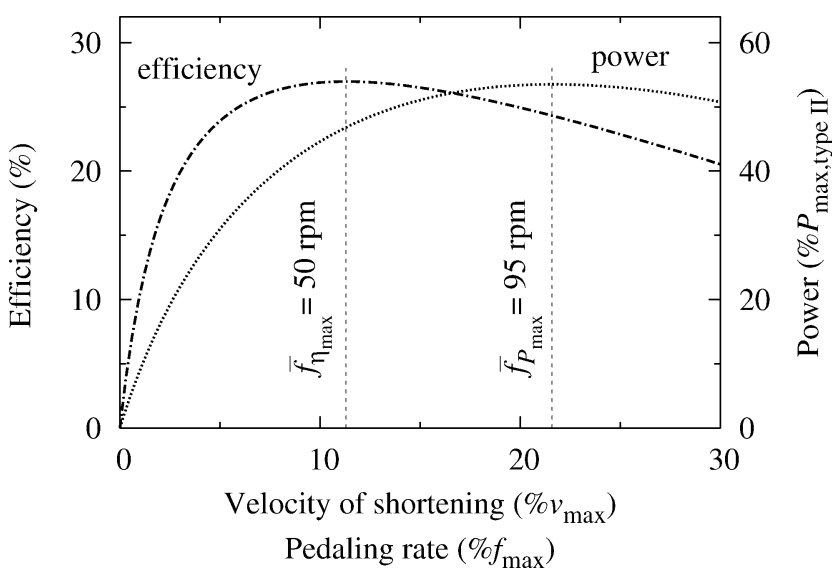

Fig. 7 Efficiency and relative power $\left(\% P_{\max }\right)$ of mixed muscles (cross-sectional area: $50 \%$ type I, $50 \%$ type II) for varying shortening velocities (or pedaling rates), see also Figs. 2 and 4. When the most efficient pedaling rate $\left(f_{\eta \max }\right)$ is $50 \mathrm{rpm}$, the most powerful pedaling rate $\left(f_{P \max }\right)$ is $95 \mathrm{rpm}$. The most efficient pedaling rate generates less power than the most powerful pedaling rate at a given cross-sectional area. At the most powerful pedaling rate, efficiency is lower than at the most efficient pedaling rate 
EMG activity for a workload of $400 \mathrm{~W}$ had been measured at a cadence of $100 \mathrm{rpm}$ (Macintosh et al. 2000).

It now becomes evident why a cyclist cannot produce maximum power at the most efficient pedaling rate: the most efficient pedaling rate yields less power for a given cross-sectional area than the most powerful pedaling rate (Fig. 7). Hence, a cyclist cannot produce the greatest power by using the most efficient pedaling rate.

It should be pointed out here that Eq. 12 is a correct description of efficiency if, and only if, the energy source for work performance is the enthalpy change of the appropriate reactions $(\Delta H)$. In the case of biological engines, such as the muscle, the source for work performance is the free energy change, $(\Delta G=\Delta H-T \Delta S)$, where $T$ is the absolute temperature and $\Delta S$ is the entropy change as the reagents are transformed into products. However, for oxidative processes, $\Delta H$ and $\Delta G$ are approximately equal, so the efficiency, as obtained from Eq. 12 , is essentially equal to the "true" thermodynamic efficiency of muscle contraction (Wilkie 1960).

In our consideration, the important point is the most efficient shortening velocity rather than peak efficiency and the progression of efficiency related to shortening velocity. Eq. 18 shows that the most efficient shortening velocity is not affected as long as the second term of the denominator in Eq. 12 is proportional to the heat released (Eq. 13). Thus, our calculations result in a more general meaning.

What is the most advantageous pedaling rate for cyclists? Using the most powerful pedaling rate results in a higher energy turnover at a given workload than using the most efficient pedaling rate (Fig. 7). In competitions of short duration, the energy aspect may not be important. In a long-distance race, however, the importance of energy supply increases: using the most powerful pedaling rate may result in early exhaustion of the cyclist. In track cycling and sprints, cadences of $120 \mathrm{rpm}$ and more are observed because the duration of the race is rather short, and the most powerful pedaling rate is more advantageous. In very long-distance races, low cadences between 50 and $60 \mathrm{rpm}$ are preferred because energy uptake and turnover rate is limited, and using the most efficient pedaling rate is more advantageous than using the most powerful one.

Energy can be supplied aerobically and/or anaerobically. Additionally, energy supply is not the only parameter determining a cyclist's performance. The fiber-type proportion and muscle fatigue are also involved. In mathematical terms, the most advantageous pedaling rate is a function of many variables. Unfortunately, these variables are not independent: energy supply and the used fiber-type proportion depend on fatigue and workload, fatigue depends on workload and duration etc. Therefore, it would be too simple to state that the most advantageous cadence decreases continuously in a linear manner from the most powerful to the most efficient pedaling rate as the race duration increases. As the most advantageous cadence is a function of many variables, it is difficult to discuss variations of all variables at once. It is much easier to discuss the variation of one single variable, and keep the others constant.

In sprints, energy can be supplied anaerobically resulting in a high lactic acid production within the muscle. During short race durations or in sprints at the end of a competition, this accumulation might not be a limiting factor. But, as the race duration increases, less energy can be supplied anaerobically. If energy supply must be aerobic to prevent lactic acid accumulation, and if fatigue does not play any role, the most advantageous pedaling rate is the most efficient one: most mechanical power can be produced at a given energy turnover rate, which is limited by the aerobic capacity. This condition seems to be at a duration between 20 and $30 \mathrm{~min}$. Therefore, most test protocols for the determination of the maximum lactate steady state use this time period (Beneke 2003). Hallén (personal communications) has recently observed that cyclists are fastest in a 30-min time trial using a more efficient rather than a more powerful pedaling rate.

The influence of fatigue on the pedaling rate is highly complex. Fatigue has several aspects: it can be local or central, and its mechanisms are still poorly understood. If local muscular fatigue is the most important limiting factor, a pedaling rate higher than the most efficient one might be the most advantageous one: less muscle crosssectional area is needed for a given workload than using the most efficient one. Thus, each muscle fiber is used for a shorter time period, and has more time for recovery. Therefore, using a pedaling rate higher than the most efficient one could be a possibility for the cyclist to resist muscular fatigue even if the total energy turnover is higher due to a lower efficiency. This explains the observation that using the free-chosen pedaling rate gives higher endurance performance in a 1-h time trial than using a lower more efficient pedaling rate (Nielsen et al. 2004).

There is no doubt that the most advantageous pedaling rate for a competitive cyclist is between the most efficient and the most powerful cadence. The various involved parameters complicate the choice of the most advantageous pedaling rate. Metabolic energy supply, fatigue, and the proportion of activated fiber types change dynamically during a race. Additionally, the profile of a competition is not always flat. In each race, the competitor needs to find the most advantageous balance between efficiency, power, and fatigue.

According to our calculations, the most powerful and the most efficient pedaling rates depend on the proportion of the activated cross-sectional areas of the two fiber types. The greater the proportion of type II fibers, the higher the pedaling rates for maximum power and maximum efficiency (Fig. 3). We assume that higher exercise power leads to the recruitment of a greater proportion of activated type II fibers. Hence, the most 


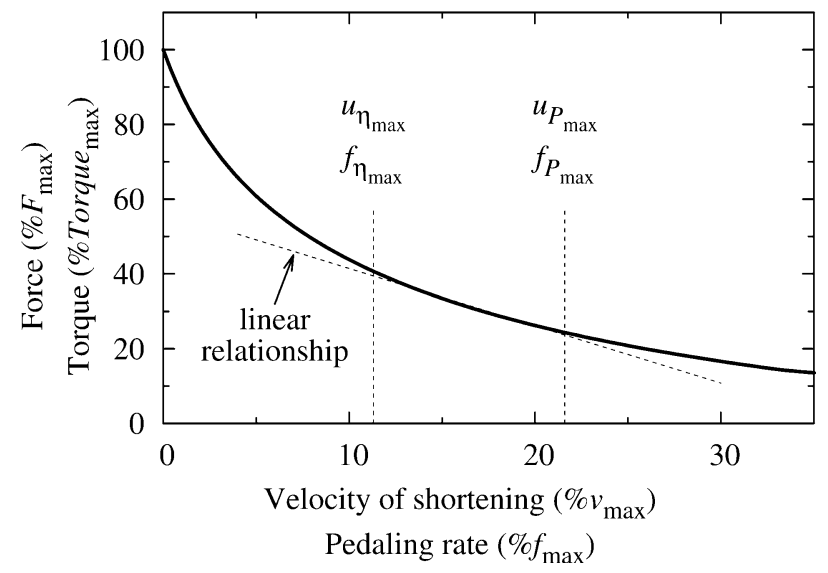

Fig. 8 Detail from the hyperbolic force-velocity realtionship (see Fig. 1) for mixed muscle with equal cross-sectional areas of both fiber types. In the range between $u_{\eta \max }, f_{\eta_{\max }}$ and $u_{P \max }, f_{P_{\max }}$, the relationship is almost linear. When $\left.f_{\eta \max }\right)$ is $\left.50 \mathrm{rpm}, f_{P \max }\right)$ is $95 \mathrm{rpm}$

efficient pedaling rate increases when power increases. Laboratory observations confirm this assumption (di Prampero 2000). Furthermore, the cadence that produces the lowest EMG activity at a given workload should increase with the workload, as experimentally observed by Macintosh et al. (2000).

Hill's (1938) force-velocity relationship was found in vitro in isolated muscle preparations. Cycling includes large muscle groups and more than one joint. Therefore, one might argue that Hill's hyperbolic equation does not apply to cycling. Moreover, a linear relationship was found between torque and crank velocity during cycling (McCartney et al. 1983; Sargeant et al. 1981; Vandewalle et al. 1987). Figure 8 shows a detail of the force-velocity relationship for mixed muscles from Fig. 1. In the range between the most efficient and the most powerful shortening velocity, the hyperbolic relationship is almost linear. On the basis of our assumptions, this also holds true for the torque-pedaling-rate relationship. The hyperbolic force-velocity relationship is based on the inherent muscle properties. Therefore, a hyperbolic relationship seems to be a more plausible assumption than a linear relationship. The torque-pedaling-rate relationship in cycling has also been previously described as a hyperbolic relationship (Sargeant 1994).

In physiological investigations, peak pedaling power is often ascertained by an incremental step test on a cycling ergometer at a given pedaling rate. Our results show that peak cycling power is a function of the used cadence. It is obvious that achieving maximum cycling power demands a pedaling rate rather near the most powerful one than near the most efficient one (as often applied!). But using a pedaling rate near the most powerful one results in an increased energy turnover compared to using a lower cadence near the most efficient one. This interaction between pedaling rate and energy has to be taken into account in cycling experiments. A higher energy turnover might result in a higher lactate production. If the length or the increase of the steps are not well chosen, the higher lactate production of a pedaling rate near the most powerful one might force the cyclist to an earlier termination of the test.

Based on our calculations and assumptions, the most efficient pedaling rate is lowest if the cross-sectional areas of both fiber types are similar (Fig. 5). However, each individual has its own muscle fiber-type distribution, which can be influenced by training. Type I fibers are less prone to fatigue than type II fibers, but as time elapses, type I fibers will also be subject to fatigue. It might be possible during a race to change the pedaling rate, and thus the proportion of activated type I and type II fibers. Thus, the choice of cadence might enable a cyclist to lower the activity of the more fatigued fibers, allowing them to temporarily recover.

So far, we have not calculated the maximal pedaling rate on the basis of $F=0$ (Fig. 1). Assuming that the most efficient pedaling rate is between 42 and $60 \mathrm{rpm}$, we obtain a theoretical value for the maximal pedaling rate of $372-531 \mathrm{rpm}$. Both the maximal muscle shortening velocity and the maximal pedaling rate depend on the geometry of the joints and the length of the muscles. Muscle length is equivalent to the number of contractile elements arranged in series. The number of contractile elements in series can increase or decrease (Williams and Goldspink 1978), as opposed to the given joint geometry of adults. A special training including stretching, which affects the number of contractile elements, might change the most efficient and the most powerful pedaling rate.

Our results enable us to explain several observations in the laboratory and in the field. Even if additional physiological influences are assumed, the inherent properties of muscles described by the force-velocity relationship (Hill 1938) and the heat release (Hill 1964) seem to be the most important factors affecting the pedaling rate of competitive cyclists.

We have used the muscle-specific constants $a_{0, \mathrm{I}}=0.15, a_{0, \mathrm{II}}=0.25$, and the ratio $V=v_{\max , \mathrm{II}} / v_{\max , \mathrm{I}}=3$, but these constants may vary individually. Our equations are sufficiently flexible to accommodate individual variations of these parameters. To facilitate calculations with changed parameters, we have written a small computer program available on request.

\section{Conclusion}

We conclude that the optimal pedaling rate, representing the fastest race performance, is not fixed but depends on race duration; it ranges between $f_{\eta \max }$ to $f_{P \max }$. Our results are not only interesting for competitive cyclists, but also for investigations using cycling ergometers: maximum power will not be reached by using a pedaling rate near the most efficient one.

Acknowledgements We acknowledge Professor Pietro di Prampero's helpful discussions during the preparation of this article. 


\section{References}

Baron R (2001) Aerobic and anaerobic power characteristics of offroad cyclists. Med Sci Sports Exerc 33:1387-1393

Beneke R (2003) Methodological aspects of maximal lactate steady state - implications for performance testing. Eur J Appl Physiol 89:95-99

Di Prampero PE (2000) Cycling on earth, in space, on the moon. Eur J Appl Physiol 82:345-360

Dorel S, Bourdin M, Van Praagh E, Lacour J-R, Hautier CA (2003) Influence of two pedalling rate conditions on mechanical output and physiological responses during all-out intermittent exercise. Eur J Appl Physiol 89:157-165

Faulkner J A, Claflin D R, McCully KK (1986) Power output of fast and slow fibers from human skeletal muscles. In: Jones NL, McCartney N, McComas AJ (eds) Human muscle power. Human Kinetics, Champaign, Ill., pp 81-94

Gotshall RW, Bauer TA, Fahrner SL (1996) Cycling cadence alters exercise hemodynamics. Int J Sports Med 17:17-21

Hill AV (1938) The heat of shortening and the dynamic constants of muscle. Proc R Soc Lond 126:136-195

Hill AV (1964) The effect of load on the heat of shortening of muscle. Proc R Soc Lond 159:297-318

Macintosh BR, Neptune RR, Horton JF (2000) Cadence, power, and muscle activation in cycle ergometry. Med Sci Sports Exerc $32: 1281-1287$
Martin JC, Spirduso WW (2001) Determination of maximal cycling power: crank length, pedaling rate and pedal speed. Eur J Appl Physiol 84:413-418

McCartney N, Heigenhauser GJF, Jones NL (1983) Power output and fatigue of human muscle in maximal cycling exercise. J Appl Physiol 55:218-224

Nielsen JS, Hansen EA, Sjøgaard G (2004) Pedalling rate affects endurance performance during high-intensity cycling. Eur J Appl Physiol 92:114-120

Sargeant AJ (1994) Human power output and muscle fatigue. Int J Sports Med 15:116-121

Sargeant AJ, Hoinville E, Young A (1981) Maximum leg force and power output during short-term dynamic exercise. J Appl Physiol 51:1175-1182

Vandewalle H, Pérès G, Heller J, Panel J, Monod H (1987) Forcevelocity relationship and maximal power on a cycle ergometer. Eur J Appl Physiol 56:650-656

Wendt IR, Gibbs CL (1979) Recovery heat production of mammalian fast- and slow-twitch muscles. Am J Physiol 230:1637-1643

Widrick JJ, Freedson PS, Hamill J (1992) Effect of internal work on the calculation of optimal pedaling rates. Med Sci Sport Exerc 24:376-382

Wilkie DR (1960) Thermodynamics and the interpretation of biological heat measurements. Prog Biophys Mol Biol 10:259-298

Williams PE, Goldspink G (1978) Changes in sarcomer length and physiological properties in immobilized muscle. J Anat 127:459-468 